BULL. AUSTRAL. MATH. SOC.

VOL. $3(1970), 273-276$.

\title{
A solvability condition for finite groups with nilpotent maximal subgroups
}

\author{
John Randolph
}

Let $G$ be a finite group with a nilpotent maximal subgroup $S$ and let $P$ denote the 2-Sylow subgroup of $S$. It is shown that if $P \cap Q$ is a normal subgroup of $P$ for any 2-Sylow subgroup $Q$ of $G$, ther $G$ is solvable.

Thompson [6] has shown that if a finite group $G$ has a nilpotent maximal subgroup $S$ of odd order, then $G$ is solvable. Janko [3] has extended this result by proving that if the 2-Sylow subgroup of $S$ has class $\leq 2$, then $G$ is solvable. We note here another condition under which $G$ is solvable.

THEOREM. Let $G$ be a finite group with a nilpotent maximal subgroup $S$ and let $P$ denote the 2-Sylow subgroup of $S$. Suppose that given any 2-Sylow subgroup $Q$ of $G, P \cap Q$ is a normal subgroup of $P$. Then $G$ is solvable.

Proof. Assume the theorem is false and let $G$ be a minimal counterexample. Let $\operatorname{rad}(G)$ denote the radical of $G$ (the largest solvable normal subgroup of $G$ ) and distinguish two cases.

Case 1. $\operatorname{rad}(G)=1$. Then $G$ has a non-abelian minimal normal subgroup $M$.

LEMMA [4]. If a finite group $G$ contains a nilpotent maximal subgroup $S$ and a non-abelian minimal normal subgroup $M$ and if $P$ is

Received 30 June 1970. 
the 2-Sylow subgroup of $S$, then $S=\operatorname{Prad}(G), G=\operatorname{MPrad}(G)$ and $P$ is a 2-Syzow subgroup of $G$.

By the lemma, $G=M P, P$ is a 2-Sylow subgroup of $G$ and $P$ is a maximal subgroup of $G$. Let $R$ be a 2-Sylow subgroup of $G$ such that $R \neq P$. If $R \cap P \neq I$, then $P \subseteq N_{G}(R \cap P)$ by hypothesis. Write $R=g P g^{-1}$ for some $g$ in $G \cdot P \cap g P g^{-1}$ is normal in $P$ so $g\left(P \cap g^{-1} P_{g}\right) g^{-1}=R \cap P \quad$ is normal in $R$. Hence $\langle R, P\rangle \subseteq N_{G}(R \cap P)$. But $P$ is maximal in $G$ so $R \cap P$ is normal in $G$, contradicting $\operatorname{rad}(G)=1$. Thus $R \cap P=1$, and from Theorem 1.4, p. 302 of [2], we conclude that $G$ has exactly one conjugate class of involutions.

By [6], $P$ has at least one involution. Suppose that $P$ has more than one involution. Let $a$ be an involution in the center of $P$ and pick an involution $b$ in $P$ such that $a \neq b$. Let $A=\{a\}$ and $B=\{b\}$. Since $G$ has only one conjugate class of involutions, $A$ and $B$ are subsets of $P$ conjugate in $G$, and Corollary 2.7, p. 245 of [2], implies that there exist 2-Sylow subgroups $Q_{i}$ of $G$ and subsets $A=A_{0}, A_{1}, \ldots, A_{m}=B$ of $P$ such that $A_{i} \subseteq P \cap Q_{i}$ and $A_{i}=A_{i-1}^{y_{i}}$ for some $y_{i}$ in $N_{G}\left(P \cap Q_{i}\right), 1 \leq i \leq m$. Thus $P \cap Q_{i} \neq 1$ and since $P$ is disjoint from its distinct conjugates, $P=Q_{i}$ and $N_{G}\left(P \cap Q_{i}\right)=N_{G}(P)$. However $P$ is maximal in $G$ and $\operatorname{rad}(G)=1$, so $N_{G}(P)=P, y_{i}$ is in $P$ for each $i$, and $b$ is conjugate to $a$ in $P$, a contradiction. Therefore $P$ has exactly one involution.

Since $M$ is a non-abelian minimal normal subgroup of $G$, we may write $M=M_{1} \times \ldots \times M_{t}, t \geq 1$, where the $M_{i}$ are mutually isomorphic non-abelian simple groups, by Theorem 4.4 .4 of [5]. Since $P$ is a 2-Sylow subgroup of $G$ and $M_{i}$ is a subnormal subgroup of $G, M_{i} \cap P$ is a 2-Sylow subgroup of $M_{i}$. $M_{i}$ cannot be solvable, so $M_{i} \cap P \neq 1$ by the Feit-Thompson theorem. Thus $M_{i} \cap P$ has exactly one involution and is therefore cyclic or generalized quaternion, by Theorem 9.7.3 of [5]. If $M_{i} \cap P$ is cyclic then $M_{i}$ has a normal 2-complement by 
Theorem 6.2.11 of [5], a contradiction. If $M_{i} \cap P$ is generalized quaternion, [1] implies that $M_{i}$ is not simple, a contradiction. Case 1 is now eliminated.

Case 2. $\operatorname{rad}(G) \neq 1$. We assert that given any 2-Sylow subgroup $Q$ of $G, P \cap Q \operatorname{rad}(G)$ is normal in $P$. For if $P$ is normal in $G, P$ is contained in $Q$ and $P \cap Q \operatorname{rad}(G)=P$. If $P$ is not normal in $G$ then $N_{G}(P)=S$ and $P$ is a 2-Sylow subgroup of $G$. Now $Q=g P g^{-1}$ for some $g$ in $G$ and $Q \subseteq g S g^{-1}$. Since $g S g^{-1}$ is a nilpotent maximal subgroup of $G$ and $G$ is not solvable, $\operatorname{rad}(G) \subseteq g S g^{-1}$. So $Q$ is the 2-Sylow subgroup of the nilpotent group $Q \operatorname{rad}(G)$. It follows that $P \cap \operatorname{Qrad}(G) \subseteq P \cap Q$. Thus $P \cap \operatorname{Qrad}(G)=P \cap Q$, a normal subgroup of $P$ by hypothesis.

Let $\bar{G}=G / \operatorname{rad}(G), \bar{S}=S / \operatorname{rad}(G)$ and $\bar{P}=\operatorname{Prad}(G) / \operatorname{rad}(G)$. $\bar{S}$ is a nilpotent maximal subgroup of $\bar{G}$ and $\bar{P}$ is the 2-Sylow subgroup of $\bar{S}$. Let $\bar{Q}$ be any 2-Sylow subgroup of $\bar{G}$. Write $\bar{Q}=\operatorname{Qrad}(G) / \operatorname{rad}(G)$ for some 2-Sylow subgroup $Q$ of $G$. Since $P \cap \operatorname{Qrad}(G)$ is normal in $P$, $\bar{P} \cap \bar{Q}$ is normal in $\bar{P}$. Since $G$ was a minimal counterexample to the theorem and $|\bar{G}|<|G|, \bar{G}$ is solvable. Hence $G$ is solvable, a contradiction. This completes the proof of the theorem.

\section{References}

[1] Richard Brauer and Michio Suzuki, "On finite groups of even order whose 2-Sylow group is a quaternion group", Proc. Nat. Acad. Sci. U.S.A. 45 (1959), 1757-1759.

[2] Daniel Gorenstein, Finite groups (Harper's Series in Modern Mathematics, Harper \& Row, New York, Evanston, London, 1968).

[3] . Zvonimir Janko, "Finite groups with a nilpotent maximal subgroup", $J$. Austral. Math. Soc. 4 (1964), 449-451.

[4] John Randolph, "On a theorem of Thompson concerning a class of non-solvable groups", (to appear). 
[5] W.R. Scott, Group theory (Prentice-Hall, Englewood Cliffs, New Jersey, 1964).

[6] John G. Thompson, "Normal p-complements for finite groups", Math. Z. 72 (1960), 332-354.

West Virginia University,

Morgantown, West Virginia,

USA. 